\title{
Molecular basis of fruit development
}

\author{
Zhongchi Liu ${ }^{1 *}$ and Robert G. Franks ${ }^{2 *}$ \\ ${ }^{1}$ Department of Cell Biology and Molecular Genetics, University of Maryland, College Park, MD, USA \\ ${ }^{2}$ Department of Plant and Microbial Biology, North Carolina State University, Raleigh, NC, USA \\ *Correspondence: zliu@umd.edu; rgfranks@ncsu.edu \\ Edited by: \\ Kimberley Cathryn Snowden, The New Zealand Institute for Plant and Food Research Limited, New Zealand \\ Reviewed by: \\ David Smyth, Monash University, Australia
}

Keywords: fruit evolution, endocarp, auxin, cytokinin, morphogenesis, ovule, gynoecium, carpel margin meristem

The fruit is a vital plant structure that supports seed development and dispersal, and is an indispensable part of the human diet. The 11 articles within this special research topic focus on the molecular mechanisms of early fruit development and span a diversity of species and experimental approaches. Since the gynoecium, the female floral structure, is the precursor of all or part of the fruit, several articles are focused on mechanisms of gynoecium development. The articles can be organized into several groups based on common themes highlighted below.

\section{PATTERNING OF THE GYNOECIUM}

The gynoecium consists of one to several carpels, usually fused together and topped with style and stigma. The botanical fruit is derived from the carpel wall (pericarp) and genes that regulate gynoecium development ultimately affect fruit size, shape, and dispersal mode. Hence, one could not discuss fruit development without understanding the mechanism controlling the gynoecium development.

The gynoecium is a three dimensional structure with three positional axes: basal-apical; medial-lateral; and abaxial-adaxial. Auxin synthesis, transport, and signaling have been implicated in the regulation of all three axes. Previously, the auxin gradient model (Nemhauser et al., 2000) proposed that auxin was synthesized at the apical tip of the gynoecium and then transported basally, forming a gradient from high auxin concentrations at the apex to low concentrations at the base. The differential cellular responses to the auxin gradient resulted in the apical-basal patterning of the gynoecium.

In this research topic, Zuniga-Mayo et al. (2014) added a new dimension to this model by showing that exogenous cytokinin application (benzyl amino purine, BAP) to the Arabidopsis inflorescence caused a phenotype similar to that caused by the auxin transport inhibitor NPA (1-N-naphtylphtalamic acid). Hence, cytokinin may reduce auxin transport and thus also be involved in gynoecium apical-basal patterning.

Hawkins and Liu (2014) proposed an alternative model in lieu of the auxin gradient model. They pointed out that the gynoecium apical-basal axis determination likely occurred very early, long before auxin biosynthesis occurs at the apical tip. This new model suggests that, much like leaf patterning, it is the role of auxin in the abaxial-adaxial polarity establishment that determines proper apical to basal patterning of the gynoecium.
At later stages of the gynoecium development, auxin biosynthesis at the apex may be critical to formation of the style and stigma. In NGATHA (NGA) gain- or loss-of-function mutants of Arabidopsis, when apical development is disrupted, MartinezFernandez et al. (2014) identified 2449 genes whose expression levels were altered. Their analysis of these genes suggests that the NGA proteins regulate gynoecial development via the control of auxin homeostasis.

\section{CARPEL MARGIN MERISTEMS (CMMs) AND SHOOT APICAL MERISTEM (SAM) ARE REGULATED WITH SIMILAR MECHANISMS}

Carpel margin meristems (CMMs) are the meristematic medial portions of the gynoecium that give rise to the ovules. Arnaud and Pautot (2014) reviewed the roles of TALE HD (three amino acid loop extension homeodomain) transcription factors in regulating CMMs of Arabidopsis, highlighting similar molecular mechanisms underlying CMM and SAM (shoot apical meristem) with respect to gibberellic acid and cytokinin signaling. Another similarity between the CMM and the SAM was highlighted by Kamiuchi et al. (2014) as they defined the role of CUP-SHAPED COTYLEDON1 (CUC1) and CUC2 genes in initiating and positioning the CMM and in the regulation of SHOOTMERISTEMLESS (STM) expression in the gynoecium. Wynn et al. (2014) reveal a role for the transcription factor PERIANTHIA (PAN) during CMM development as well as during floral meristem determinancy. Their work suggested that proper termination of the floral meristem may be required for the complete development of the CMM and ovules.

Cucinotta et al. (2014) reviewed the early ovule development with a focus on the formation of the CMM and the initiation of ovule primordia in Arabidopsis. They presented a model of ovule initiation that relates the functions of CUC and ANT genes as well as the action of auxin, cytokinin, and brassinosteroid hormones (Galbiati et al., 2013; Cucinotta et al., 2014). Their model posits a role for $A N T$ in the growth of the organ primordia, and CUC genes in the specification of the boundary zones between ovules. The interactions between the primordial and boundary regions, as well as cytokinin regulation are required for the proper expression and localization of the PIN-FORMED1 (PIN1) auxin transporter and thus for 
proper auxin fluxes. Brassinosteroid signaling is proposed to support ovule initiation through the stimulation of ANT activity in ovule primordia (Huang et al., 2013; Cucinotta et al., 2014).

\section{FRUIT SHAPE, SIZE, AND RIPENING}

Two articles each provided a unique perspective on fruit development and ripening. Van Der Knaap et al. (2014) discussed six key genes and their mechanisms that regulate tomato fruit shape and weight. Some of the genes also act during floral meristem and floral organ development, highlighting the close connection between floral organ initiation, specification and later fruit shape and sizes.

The review by Pesaresi et al. (2014) focused on the retrograde (plastids to nucleus) and anterograde (nucleus to plastids) communication pathways during fruit ripening, areas that were not yet fully explored. In the anterograde pathway, nuclear-encoded regulators alter plastid function and specify plastid types (Leon et al., 1998; Raynaud et al., 2007). The retrograde pathways allow for the transfer of information from the plastid to the nucleus regarding the functional or physiological status of the plastids (Chi et al., 2013).

\section{EVOLUTIONARY PERSPECTIVES OF FRUIT DEVELOPMENT}

Upon fertilization, the carpel in many species transitions from an ovule-containing vessel to the seed containing fruit. Dardick and Callahan (2014) reviewed molecular mechanisms that regulate endocarp differentiation in each of three species from the families Brassicaceae, Rosaceae, and Solanaceae. The endocarp is the innermost cell layer of the carpel wall. They discussed in detail current understanding of the "stone" endocarp in peach and suggested that the regulatory genes and pathways controlling the lignified valve margin layer of Brassica's dry fruit are similar to those controlling lignified "stone" endocarp in peach.

Pabon-Mora et al. (2014) took a phylogenetic approach to analyze the transcription factor genes that regulate carpel valve margins of dry fruit in Arabidopsis. Through comprehensive searches for homologs across core-eudicots, basal eudicots, monocots, and basal angiosperms and phylogenetic tree construction, the authors suggested conservation of certain fruit development pathways and established the foundation for future functional tests.

\section{SUMMARY}

The diverse perspectives presented in this research topic provide an in depth understanding of ongoing researches in this exciting and evolving field. One common theme emerging from several articles is that distinct structures do not always result from entirely distinct regulatory networks, (e.g., similar genes regulate SAM, FM, and CMM development, similar mechanisms may underlie patterning of carpels and leaves, and conserved networks are required for the stone endocarp in peach and the dry fruit valve margin in Brassica). Because of the broad biological questions addressed as well as the potential applications to agricultural problems, this field will likely attract further interest and funding, and yield important discoveries in the future.

\section{ACKNOWLEDGMENTS}

We would like to thank Dr. Cristina Ferrandiz for the cover image. Work in the laboratories of Zhongchi Liu and Robert Franks has been funded by the National Science Foundation (MCB0951460 and MCB0923913 to Zhongchi Liu and IOS1355019 to Robert G. Franks).

\section{REFERENCES}

Arnaud, N., and Pautot, V. (2014). Ring the BELL and tie the KNOX: roles for TALEs in gynoecium development. Front. Plant Sci. 5:93. doi: $10.3389 /$ fpls.2014.00093

Chi, W., Sun, X., and Zhang, L. (2013). Intracellular signaling from plastid to nucleus. Annu. Rev. Plant Biol. 64, 559-582. doi: 10.1146/annurev-arplant050312-120147

Cucinotta, M., Colombo, L., and Roig-Villanova, I. (2014). Ovule development, a new model for lateral organ formation. Front. Plant Sci. 5:117. doi: $10.3389 /$ fpls.2014.00117

Dardick, C., and Callahan, A. M. (2014). Evolution of the fruit endocarp: molecular mechanisms underlying adaptations in seed protection and dispersal strategies. Front. Plant Sci. 5:284. doi: 10.3389/fpls.2014.00284

Galbiati, F., Sinha Roy, D., Simonini, S., Cucinotta, M., Ceccato, L., Cuesta, C., et al. (2013). An integrative model of the control of ovule primordia formation. Plant J. 76, 446-455. doi: Doi 10.1111/Tpj.12309

Hawkins, C., and Liu, Z. (2014). A model for an early role of auxin in Arabidopsis gynoecium morphogenesis. Front. Plant Sci. 5:327. doi: 10.3389/fpls.2014. 00327

Huang, H. Y., Jiang, W. B., Hu, Y. W., Wu, P., Zhu, J. Y., Liang, W. Q., et al. (2013). BR signal influences Arabidopsis ovule and seed number through regulating related genes expression by BZR1. Mol. Plant 6, 456-469. doi: $10.1093 / \mathrm{mp} / \mathrm{sss} 070$

Kamiuchi, Y., Yamamoto, K., Furutani, M., Tasaka, M., and Aida, M. (2014) The CUC1 and CUC2 genes promote carpel margin meristem formation during Arabidopsis gynoecium development. Front. Plant Sci. 5:165. doi: 10.3389/fpls.2014.00165

Leon, P., Arroyo, A., and Mackenzie, S. (1998). Nuclear control of plastid and mitochondrial development in higher plants. Annu. Rev. Plant Physiol. Plant Mol. Biol. 49, 453-480. doi: 10.1146/annurev.arplant. 49.1.453

Martinez-Fernandez, I., Sanchis, S., Marini, N., Balanza, V., Ballester, P., NavarreteGomez, M., et al. (2014). The effect of NGATHA altered activity on auxin signaling pathways within the Arabidopsis gynoecium. Front. Plant Sci. 5:210. doi: $10.3389 /$ fpls.2014.00210

Nemhauser, J. L., Feldman, L. J., and Zambryski, P. C. (2000). Auxin and ETTIN in Arabidopsis gynoecium morphogenesis. Development 127, 3877-3888.

Pabon-Mora, N., Wong, G. K., and Ambrose, B. A. (2014). Evolution of fruit development genes in flowering plants. Front. Plant Sci. 5:300. doi: 10.3389/fpls.2014.00300

Pesaresi, P., Mizzotti, C., Colombo, M., and Masiero, S. (2014). Genetic regulation and structural changes during tomato fruit development and ripening. Front. Plant Sci. 5:124. doi: 10.3389/fpls.2014.00124

Raynaud, C., Loiselay, C., Wostrikoff, K., Kuras, R., Girard-Bascou, J., Wollman, F. A., et al. (2007). Evidence for regulatory function of nucleusencoded factors on mRNA stabilization and translation in the chloroplast. Proc. Natl. Acad. Sci. U.S.A. 104, 9093-9098. doi: 10.1073/pnas. 0703162104

Van Der Knaap, E., Chakrabarti, M., Chu, Y. H., Clevenger, J. P., Illa-Berenguer, E., Huang, Z., et al. (2014). What lies beyond the eye: the molecular mechanisms regulating tomato fruit weight and shape. Front. Plant Sci. 5:227. doi: 10.3389/fpls.2014.00227

Wynn, A. N., Seaman, A. A., Jones, A. L., and Franks, R. G. (2014). Novel functional roles for PERIANTHIA and SEUSS during floral organ identity specification, floral meristem termination, and gynoecial development. Front. Plant Sci. 5:130. doi: 10.3389/fpls.2014.00130 
Zuniga-Mayo, V. M., Reyes-Olalde, J. I., Marsch-Martinez, N., and De Folter, S. (2014). Cytokinin treatments affect the apical-basal patterning of the Arabidopsis gynoecium and resemble the effects of polar auxin transport inhibition. Front. Plant Sci. 5:191. doi: 10.3389/fpls.2014.00191

Conflict of Interest Statement: The authors declare that the research was conducted in the absence of any commercial or financial relationships that could be construed as a potential conflict of interest.

Received: 15 December 2014; accepted: 12 January 2015; published online: 05 February 2015.
Citation: Liu $Z$ and Franks RG (2015) Molecular basis of fruit development. Front. Plant Sci. 6:28. doi: 10.3389/fpls.2015.00028

This article was submitted to Plant Evolution and Development, a section of the journal Frontiers in Plant Science.

Copyright (c) 2015 Liu and Franks. This is an open-access article distributed under the terms of the Creative Commons Attribution License (CCBY). The use, distribution or reproduction in other forums is permitted, provided the original author(s) or licensor are credited and that the original publication in this journal is cited, in accordance with accepted academic practice. No use, distribution or reproduction is permitted which does not comply with these terms. 